\title{
Vergleichende Studien über Herz- und Gefäßwirkung von Digitalispräparaten am Frosch ${ }^{1}$ ).
}

\author{
Von \\ E. P. Pick und R. Wagner.
}

(Aus dem pharmakologischen Institut der Universität Wien.)

(Eingegangen am 14. Oktober 1920.)

Die alte klinische Erfahrungstatsache, daß in vielen Fällen neben der Herzwirkung die gefäßkonstringierende Wirkung der Digitalispräparate den Hauptanteil des therapeutischen Effektes bildet, hat uns veranlaßt, zwischen der Herz- und Gefäßwirkung verschiedener Digitaliskörper vergleichende Studien anzustellen. Wenn auch naturgemäß im Vordergrund des klinischen Interesses die Herzwirkung steht, so ist doch insbesondere durch die Untersuchungen von Gottlieb, Magnus und ihren Mitarbeitern mit Sicherheit nachgewiesen worden, daß auch der Gefäßwirkung im lebenden Organismus eine nicht unwesentliche Rolle zufällt. Allerdings sagt Schmiedeberg²): „Die Digitalis und alle übrigen therapeutisch benützten Stoffe dieser Gruppe wären schlechte Mittel bei Herzkrankheiten, wenn sie die kleineren Arterien und vielleicht auch die Capillaren enger machten und dem Durchtritt des Blutes einen größeren Widerstand bereiteten." Er kommt auf rechnerischem Wege unter Zugrundelegung der experimentellen Untersuchungen von Fahrenkamps), indem er die Beweiskraft der an überlebenden tierischen Organen erhobenen Gefäßwirkung der Digitaliskörper bezweifelt, zu dem Schlusse, daß eine „Einwirkung der therapeutisch gebräuchlichen Gaben dieser Stoffe auf die Gefäßweite an Gesunden und Kranken nicht nachweisbar ist." Wie dem auch sei, die Gefäßwirkung der Digitaliskörper an überlebenden Organen stellt zweifellos neben der Herzwirkung einen Hauptfaktor der gesamten Digitaliswirkung dar, welche geeignet ist, als physiologisches Charakteristikum der verschiedenen Präparate zu dienen. Bislang erfolgte die physiologische Auswertung und Charakterisierung ausschließlich an Herzpräparaten. Nichtsdestoweniger ișt es sowohl für den Kliniker wie für den Pharmakologen von großem Interesse, ein Urteil über die quantitativen Verhältnisse der Gefäßwirkungen

\footnotetext{
1) Ausgeführt mit Unterstützung der Fürst-Liechtenstein-Spende.

2) Sch miedeberg, Grundriß der Pharmakologie. Leipzig. 7. Auflage. S. 319.

s) Schmiedebergs Arch. 65, 367.
} 
zu erhalten und über den Vergleich derselben zur reinen Herzwirkung.

Wir beabsichtigten daher, als Ergänzung der bisher üblichen Methode, nur letale Froschherzdosen zur Charakterisierung eines Präparates anzugeben, mit Hilfe der gleichzeitigen Auswertung am Gefäßsystem Standardwerte aufzufinden, in denen Gefäß- und Herzwirkung gleicherweise zum Ausdruck kommt. Wir waren uns von vornherein der hierbei zu überwindenden Schwierigkeiten bewußt, da ja schon frühere Untersuchungen feststellen konnten, daß die Grenzwerte am Gefäßsystem nicht so konstant sind wie am Herzpräparat. Nichtsdestoweniger geht aus allen einschlägigen Untersuchungen hervor, daß Herzwirkungen zumeist von Gefäßwirkungen begleitet sind.

Bereits Fahrenkam $\mathrm{p}^{1}$ ) hat am Warmblüterherzen und an überlebenden Warmblütergefäßgebieten (Kaninchen) vergleichende Untersuchungen angestellt, wieweit die Schwellenwerte für die Gefäßwirkungen des Digitoxins von dem Schwellenwerte seiner Herzwirkung entfernt sind, und kommt dabei zu dem Resultat, daß der Eintritt beider Wirkungen am lebenden Tier nebeneinander möglich ist, wobei allerdings betont werden muß, daß zum Vergleich hauptsächlich die erweiternde Wirkung auf die Nierengefäße herangezogen wurde.

Auch die Versuche von Jose $\mathrm{ph}^{2}$ ) sprechen im gleichen Sinne, indem sie zeigen, daß die Kaninchengefäße auf Digipurat und Strophanthin in für das Herz nicht toxischen Gaben sich schon verengern.

Da für die Auswertung der Digitalispräparate gerade das Froschherz das klassische Untersuchungsobjekt darstellt, mit dessen Hilfe es in viel exakterer Weise gelingt, die Schwellenwerte der Herzwirkung zu erfassen, so haben wir uns entschlossen, die Digitaliskörper vergleichsweise am Herzen und an verschiedenen Gefäßgebieten des Frosches zu untersuchen; da sich bekanntlich verschiedene Gefäßgebiete Giften gegenüber oft gegenteilig verhalten, haben wir unsere Versuche in einzelnen Fällen sowohl an Extremitäten- als auch an Splanchnicusgefäßen durchgeführt, wollen aber für die Fragestellung der vergleichenden Herz- und Gefäßwirkung vorläufig nur die am Laewen-Trendelen burg-Präparat gewonnenen Resultate heranziehen.

Schon frühere Untersucher haben sich mit der vergleichenden Einwirkung von Digitalisglykosiden auf Herz und Gefäße beim Frosch beschäftigt. Dabei wurde festgestellt, daß die Froschgefäße die Eigenschaft haben, nach Durchströmung mit gewissen Glykosiden, speziell mit Strophanthin, einer der ursprünglichen Konstriktion folgenden Nachwirkung zu unterliegen, die in unkontrollierbarer Weise die ursprüngliche Wirkung verstärken kann, so daß die Grenzkonzentrationen

1) l. e.

2) R. Joseph, Schmiedebergs Arch. 73, 81. 
bei wiederholter Durchströmung mit verschiedenen Verdünnungsgraden nicht scharf genug hervortreten ${ }^{1}$ ). Infolgedessen haben wir in analoger Weise, wie dies bei der Auswertung der Grenzkonzentration für das Herz nötig ist, auch bei der Auswertung am Gefäßsystem für jede frische Konzentration der untersuchten Glykosidlösungen stets ein neues Gefäßpräparat verwendet; nur auf diese Weise gelingt es, die Erscheinungen der Nachwirkung auszuschalten und zu verwertbaren Grenzwerten zu gelangen. Die Gefäßwirkung der Glykoside scheint sich insofern prinzipiell verschieden von der Herzwirkung zu verhalten, als die Herzwirkung der Digitalisglykoside bei großen Konzentrationen praktisch irreversibel ist, während nach den Angaben

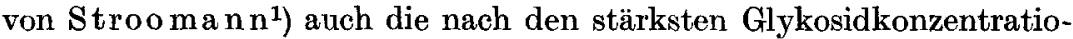
nen eingetretenen Gefäßkonstriktionen reversibel bleiben. Freilich ist auch die Vorbedingung für dieses Ergebnis jedwede Vermeidung der Entstehung eines Ödems, welches bei längerer Durchströmung in der Regel auftritt.

Wir hofften, auf diese Weise verschiedene Digitalispräparate viel schärfer charakterisieren zu können, als es bisher mittels Froschherzdosen möglich war, indem wir die Herzschwellenwerte mit den Gefäßwerten verglichen. Die Ergebnisse der in der Literatur vorliegenden Untersuchungen führten zum Schluß, daß im allgemeinen die Empfindlichkeit der Froschgefäße bei weitem die Empfindlichkeit des Herzens übertrifft; dies gilt vor allem vom Digitoxin, für welches nach den Untersuchungen von $\mathrm{Ulm}^{2}$ ) die Froschgefäße etwa hundertmal empfindlicher sind als das Froschherz. Zu demselben Schlusse kam Stroomann (l. c.) für das Digitalin, während er Strophanthin in Ubereinstimmung mit Kasztan am Herzen wirksamer fand. Ebenso fand $\mathrm{Moog}^{3}$ ), daß die Gefäßwirkung des Strophanthins g an Fröschen eine zehnmal schwächere ist als die des Digitoxins, während die Wirkung auf das isolierte Herz eine gleich starke ist; auch soll nach $\mathrm{ihm}$ das Digipurat in den schon deutlich gefäßverengernden Dosen auf das Froschherz noch keinen Einfluß haben.

Auf diese Weise läßt sich aus den im biologischen Versuch ermittelten Sehwellenwerten ein Faktor $(c / v)$ berechnen, in welchem der Zähler $c$ die am Herzen wirksame Minimalkonzentration, $v$ jene am Gefäßsystem darstellt. Es war festzustellen, in welcher Weise sich die verschiedenen Digitaliskörper in die drei möglichen Gruppen einordnen lassen, nämlich solche, in denen der Faktor $=1$, größer

$\left.{ }^{1}\right)$ H. Stroomann, Studien über die Gefäßwirkung der Digitaliskörper. Zeitschr. f. d. ges. exp. Med. 2, 278. 1914.

$\left.{ }^{2}\right)$ Marcel Ulm, Beiträge zur Gefäß- und Herzwirkung des Digitoxins am Frosch. Inaug.-Diss. Heidelberg. München 1912.

3) Moog, Beitrag zur Gefäß- und Herzwirkung des Strophanthins und des Extr. digital. depur. am Frosch. Inaug.-Diss. Köln 1912. 
oder kleiner als 1 ist. In zwei Fällen, und zwar beim Digalen Cloetta und Infus. fol. digit., konnte die Auswertung der vasokonstriktorischen Wirkung nicht derart durchgeführt werden, daß Grenzwerte erreicht wurden, da das Infusum bekanntlich reichlich vasokonstriktorische Saponinsubstanzen enthält, die die Auswertung im TrendelenburgPräparat stören.

Wir verwendeten die folgenden Präparate: Strophanthin Thoms, Strophanthin Böhringer, Digipurat Knoll, Cymarin Bayer \& Co., Digifolin „Ciba“, Infus. fol. digital wässerig, Digitoxin solub. Merck, Digalen Cloetta, Verodigen Böhringer in Tabletten à $0,1^{1}$ ).

\section{Methodik.}

Die Auswertung der Digitalispräparate am Herzen wurde am Straubschen Herzpräparat durchgeführt, wobei nicht nur auf die typische systolische Wirkung geachtet, sondern auch der diastolische Stillstand verzeichnet wurde. Die Gefäßwirkung wurde gewöhnlich am Läwen-Trendelenburgschen Froschpräparat, in einzelnen Fällen auch an dem Splanchnicusgefäßgebiet des Frosches nach der von Fröhlich angegebenen Methode und am isolierten Leberkreislauf geprüft. Hier ist hervorzuheben, daß die genaue Auswertung an den Gefäßpräparaten nur dann möglich ist, wenn die in ihm selbst liegenden Schwankungen sicher ausgeschaltet sind. $\mathrm{Zu}$ diesem Zwecke mußte jedes Gift an mehreren Froschpräparaten ausgewertet werden, wobei als Grenzwert diejenige Verdünnung angenommen wurde, welche imstande war, den sonst konstant bleibenden Ausfluß (Tropfenzahl pro Minute gezählt) innerhalb 10-15 Minuten um mindestens 30\% des Ausgangswertes der Tropfenzahl herabzudrücken. Die nächst höhere Verdünnung mußte schon unwirksam sein. Es sei nochmals bemerkt, daß für jede neue Verdünnung der Giftlösung ein neues Froschpräparat benützt worden war, um die durch ein allfälliges, fast immer bei längerer Durchströmung sich einstellendes Ödem entstehenden Störungen zu vermeiden und die sich aus der Nachwirkung der Gifte (Hysterese) ergebenden Auswertungsschwierigkeiten zu umgehen. Jedes Präparat mußte naturgemäß in zahlreichen Versuchen ausgewertet werden.

Als Versuchstier diente ausschließlich Rana esculenta. Die Versuche erstreckten sich über das ganze Jahr.

\section{Versuchsergebnisse.}

Hier sollen der Utbersicht wegen nur die tabellarisch zusammengestellten Ergebnisse wiedergegeben werden.

1) Eine Tablette wurde pulverisiert, in $1 \mathrm{ccm} 95$ proz. Alkohol gelöst und mit Ringerlösung auf die gewünschte Endkonzentration gebracht. 
Tabelle I.

\begin{tabular}{|c|c|c|c|c|c|}
\hline 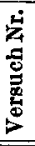 & $\begin{array}{l}\text { Name } \\
\text { des Praparates }\end{array}$ & $\begin{array}{l}\text { Minimalkonz. } \\
\text { für das Herz }{ }^{\text {) }}\end{array}$ & $\begin{array}{c}\text { Minimalkonz. } \\
\text { am Gefäß- } \\
\text { system (Läwen- } \\
\text { Trendelenburg) }\end{array}$ & $\begin{array}{c}\text { Faktor } \\
\mathbf{c} / v\end{array}$ & Anmerkung \\
\hline 1 & $\begin{array}{l}\text { Strophanthin (Kom- } \\
\text { bé) Böhringer }\end{array}$ & $1: 550000$ & $1: 300000$ & 1,83 & $\begin{array}{l}\text { diastolischer Still- } \\
\text { stand }\end{array}$ \\
\hline 2 & $\begin{array}{l}\text { Strophanth. (Gratus) } \\
\text { Thoms }\end{array}$ & $1: 300000$ & $1: 200000$ & 1,5 & $\begin{array}{l}\text { diastolischer Still- } \\
\text { stand }\end{array}$ \\
\hline 3 & Digipurat Knoll & $1: 50000$ & $1: 50000$ & 1,0 & $\begin{array}{l}\text { deutlich diastolische } \\
\text { Wirkung }\end{array}$ \\
\hline 4 & Cymarin Bayer & $1: 10000$ & $1: 15000$ & $C, 67$ & $\begin{array}{l}\text { systolischer Still- } \\
\text { stand }\end{array}$ \\
\hline 5 & Digifolin Ciba & $1: \quad 1000$ & 1: $\quad 500$ & 2,0 & $\begin{array}{l}\text { diastolischer Still- } \\
\text { stand }\end{array}$ \\
\hline 6 & Digitox. sol. Merck & $1: 450000$ & $1: 500000$ & 0,9 & Mittelstellung \\
\hline 7 & $\begin{array}{c}\text { Verodigen } \\
\text { ringer" }\end{array}$ & 1: $\quad 1750$ & $1: \quad 1250$ & 1,4 & systolische Wirkung \\
\hline 8 & Digalen Cloetta & $1: 166666$ & $1: 83333$ & 2,0 & systolische Wirkung \\
\hline
\end{tabular}

Die angeführten Untersuchungen zeigen, daß die von uns untersuchten Digitalispräparate sich in zwei Gruppen einteilen lassen: Die erste Gruppe umfaßt Präparate, welche in Ubereinstimmung mit den Untersuchungen von Gottlieb, Magnus und der Gottliebsehen Schule vorwiegend Gefäßwirkung aufweisen; zu diesen Präparaten gehört vor allem das intensivst gefäßkonstringierende Cymarin Bayer, dem sich in seiner gefäßverengernden Wirkung das Digitoxin anschließt, dann folgen die Präparate Digipuratum Knoll, dessen Herz- und Gefäßwirkung beim Frosch in den gleichen Konzentrationen auftritt. Die zweite Gruppe umfaßt die Präparate: Strophanthin Thoms und Böhringer, Digifolin Ciba, Verodigen Böhringer und Digalen Cloetta, bei welchen die Herzwirkungen in verschiedener Stärke, aber meist um eine bedeutende Größe, die Gefäßwirkung übertreffen. Haben wir also bei der ersten Gruppe es mit Substanzen zu tun, bei denen eine Gefäßwirkung ohne Herzwirkung vorkommt, so enthält die zweite Gruppe Präparate, bei denen eine Herzwirkung auch ohne Gefäßwirkung möglich ist und bei denen stets eine Gefäßwirkung auch von der Herzwirkung begleitet sein muB.

Unsere Zahlen sind gewonnen durch Auswertung an den HautMuskelgefäßen des Frosches. Wir selbst haben uns in anderweitigen Versuchen davon überzeugt, daß auch die Gefäße des gesamten Gebietes des Splanchnicus auf Digitalispräparate in gleicher Weise reagieren wie das Trendelenburgsche Präparat, so da $B$ wir in der Reaktion der letzteren Gefäße einen Anhaltspunkt erhalten zu haben glauben auch für die Reaktionsfähigkeit der übrigen Gefäßgebiete im großen

1) Alle Werte sind auf Trockensubstanz bezogen. 
und ganzen; denn der besonderen Reaktionsfähigkeit einiger kleiner Gefäßgebiete des Frosches, wie z. B. der Nierengefäße, kommt bezüglich der Auswertung des gesamten Gefäßgebietes des Splanchnicus kaum eine Rolle zu.

Tabelle II enthält eine ṫbersicht über die untersuchten Digitaliskörper, geordnet nach der Größe des Quotienten $c / v$, wobei der kleinste Quotient der stärksten Gefäßwirkung entspricht, der Quotient 1 diejenigen Präparate umfaßt, bei denen die Gefäß- und Herzwirkung gleich ist, während die Quotienten größer als 1 jenen Präparaten angehören, deren Herzwirkung über die Gefäßwirkung überwiegt.

Tabelle II.

\begin{tabular}{|c|c|}
\hline Präparat & Faktor $c^{\prime} v$ \\
\hline Cymarin Bayer . . & 0,67 \\
\hline Digitoxin sol. Merk . . & 0,90 \\
\hline Digipurat Knoll . . . . & 1,0 \\
\hline Verodigen Böhringer . . & 1,4 \\
\hline Strophanthin Thoms . . & 1,5 \\
\hline Strophanthin Böhringer. & 1,83 \\
\hline Digalen Cloetta . . . . & 2,0 \\
\hline Digifolin Ciba . . . . . & 2,0 \\
\hline
\end{tabular}

Es braucht nicht hervorgehoben zu werden, daß die hier gewonnenen Zahlen nicht ohne weiters auf die Verhältnisse beim Säugetier und Menschen übertragen werden dürfen. Nichtsdestoweniger glauben wir, durch diese Zahlen eine schärfere Kennzeichnung der Wirkungsweise der verschiedenen Präparate zu erlangen, als sie nur durch die Auswertung am Froschherzen geliefert werden kann; und es ist durchaus möglich, daß die hier gewonnenen Zahlen eine Grundlage für eine rationelle Auswahl der Präparate bei ihrer klinischen Anwendung liefern könnten. Denn, wie schon eingangs erwähnt, steht sehr häufig im Vordergrund des klinischen Bildes die Gefäßwirkung der Digitalisglykoside, während die Herzwirkung oft im Hintergrund bleibt. Andere Zahlen zeigen jedoch, daß auch im Sinne Schmiedebergs manche Präparate zunächst nur eine Herzwirkung und erst in größeren Konzentrationen auch eine Gefäßwirkung aufweisen, daß jedoch die Gefäßwirkung erst bei den höchsten Dosen eintritt. Welcher Typus bei der klinischen Anwendung sich vorteilhafter erweisen wird, läßt sich von vornherein nicht bestimmen. Es ist zweifellos, daß bei der Auswahl der Präparate das Verhältnis der Herz- zur Gefäßwirkung in bestimmten Fällen maßgebend ist und daher bei der Auswertung der Digitalispräparate die Bestimmung des Quotienten $c / v$ ein neues wichtiges Merkmal nicht nur für die unbedingt notwendige quantitative Charakterisierung, sondern auch für die qualitative Erkennung der einzelnen Präparate liefert. 